\title{
Artificial Immune Algorithm for Practical Power Economic Dispatch Problems
}

\author{
C. L. Chiang
}

\begin{abstract}
This paper proposes an artificial immune algorithm with a multiplier updating method (AIA-MU) for practical power economic dispatch (PED) considering units with prohibited operating zones (POZ). The AIA equipped with a migration operation can efficiently search and actively explore solutions. The multiplier updating (MU) is introduced to handle the system constraints. To show the advantages of the proposed algorithm, two examples are investigated, and the computational results of the proposed method are compared with that of the previous methods. The proposed approach integrates the AIA and the MU, revealing that the proposed approach has the following merits - ease of implementation; applicability to non-convex fuel cost functions of the POZ; better effectiveness than previous methods, and the requirement for only a small population in applying the optimal PED problem of generators with POZ.
\end{abstract}

Index Terms-Power economic dispatch, artificial immune algorithm, prohibited operating zones.

\section{INTRODUCTION}

Power generators may possess some POZ between their minimum and maximum generation limits, because of the practical limitations of power plant elements. Operating in those zones may cause amplification of vibrations in a shaft bearing, which must be avoided in practice. The PED problem becomes a non-convex optimization problem because the prohibited regions separate the decision space into disjoint subsets constituting a non-convex solution space [1]. Furthermore, utilities must maintain some generation capacity as spinning reserve to serve loads in the event that operating units or other equipment suddenly or unexpectedly failed.

Many approaches have been adopted to resolve such PED problems with POZ and spinning reserve. Lee et al. [1] decomposed the non-convex decision space into a small number of subsets such that each of the associated dispatch problems, if feasible, was solved via the conventional Lagrangian relaxation approach. Fan et al. [2] defined a small and advantageous set of decision space with respect to the system demand, used an algorithm to determine the most advantageous space, and then utilized the $\lambda$ - $\delta$ iterative method to find the feasible optimal dispatch solution. For infeasible solutions, they re-dispatch the units using some heuristic rules to probe the neighborhood for feasibility.

To adequately solve the dispatch problem for units with POZ and spinning reserve, solution techniques that do not

Manuscript received July 9, 2013; revised September 9, 2013. This work is financially supported by the National Science Council, Taiwan, ROC. under Grant No. NSC100-2632-E-252-001-MY3 is greatly appreciated.

C. L. Chiang is with the Department of Electronic Engineering, Nan Kai University of Technology, Taiwan, ROC (e-mail: t129r@ nkut.edu.tw). directly rely on the incremental cost function formulation but perform a direct search of the solution search space are required. Such solution techniques include: Hopfiled model of neural network [3], [4], genetic algorithm (GA) [5], and evolutionary programming (EP) [6], evolutionary strategy optimization (ESO) [7], particle swarm optimization with time varying acceleration coefficients (PSO-TVAC) [8]. Su et al. [3] employed a linear input-output model for neurons, enabling the development of an operational model for rapidly resolving the PED problems. Yalcinoz et al. [4] proposed an improved Hopfield neural network that used a slack variable technique to handle inequality constraints by mapping process for obtaining the weights and biases. Orero et al. [5] presented a penalty function approach to handle the POZ and spinning reserve constraints. Pereira-Neto et al. [7] used the ESO for solving the non-convex problem of power system. Chaturvedi et al. [8] employed the PSO-TVAC method to avoid the premature convergence for the $\mathrm{POZ}$ and spinning reserve of the PED problem.

AIA [9] is inspired by immunology, immune function and principles observed in nature. AIA is a very intricate biological system which accounts for resistance of a living body against harmful foreign entities. It is now interest of many researchers and has been successfully used in various areas of research [9], [10].

\section{SYSTEM FORMULATION}

Generally, the PED problem with some units possessing POZ can be mathematically stated as follows [11]:

$$
\text { Minimize } \quad \sum_{i=1, i \in \Omega}^{N_{p}} F_{i}\left(P_{i}\right)=\sum_{i=1}^{N_{p}} F_{i}\left(c_{i} P_{i}^{2}+b_{i} P_{i}+a_{i}\right)
$$

where $F_{i}\left(P_{i}\right)$ is the fuel cost function of the unit $i, P_{i}$ is the power generated by unit $i, a_{i}, b_{i}$, and $c_{i}$ are cost coefficients of generator $i, N_{p}$ is the number of on-line units, and $\Omega$ is the set of all on-line units. The PED problem subject to the following constraints:

\section{A. Power Balance Constraints}

The equality constraint of the power balance is given by:

$$
\sum_{i=1}^{N_{p}} P_{i}=P_{d}+P_{L}
$$

where $P_{d}$ is the system load demand, and $P_{L}$ is the transmission loss.

\section{B. System Limits}

The generating capacity constraints are written as: 


$$
P_{i}^{\min } \leq P_{i} \leq P_{i}^{\max }, \quad i=1, \cdots, n_{p}
$$

where $P_{i}^{\min }$ and $P_{i}^{\max }$ are the minimum and maximum power outputs of unit $i$.

\section{System Spinning Reserve Constraints}

Units with spinning reserve can be are given as:

$$
\begin{gathered}
\sum_{i} S_{i} \geq S_{R} \\
S_{i}=\min \left\{\left(P_{i}^{\max }-P_{i}\right), \quad S_{i}^{\max }\right\} \quad \forall i \in(\Omega-\omega)(5) \\
S_{i}=0 \quad \forall i \in \omega
\end{gathered}
$$

where $S_{i}$ is spinning reserve contribution of unit $i$ in $\mathrm{MW}, S_{R}$ stands system spinning reserve requirement in $\mathrm{MW}, P_{i}^{\max }$ is maximum generation limit of unit $i, S_{i}^{\max }$ denotes maximum spinning reserve contribution of unit $i$, and $\omega$ is set of all on-line units with prohibited zones. Due to a unit with prohibited zones may operate into one of its zones while system load is regulating, it is shown in (6) that this kind of units should not contribute any regulating reserve to the system. In other words, system spinning reserve requirement must be satisfied by way of regulating the units without prohibited zones.

\section{Units with $\mathrm{POZ}$}

The unit operating range denotes the effects of a generator with POZ [5]:

$$
\begin{array}{ll}
P_{i}^{\min } \leq P_{i} \leq P_{i, 1}^{l} \quad \text { or } \\
P_{i, j-1}^{u} \leq P_{i} \leq P_{i, j}^{l}, \quad j=2, \cdots, n_{i} \quad \text { or } \\
P_{i, n i}^{u} \leq P_{i} \leq P_{i}^{\max }, \quad \forall i \in \omega
\end{array}
$$

where $P_{i, j}^{l}$ and $P_{i, j}^{u}$ respectively are the lower and upper bounds of prohibited zone $j$ of unit $i$, and $n_{i}$ is the number of prohibited zones in unit $i$.

Clearly, the entire operating region of a dispatching unit with $n_{i}$ prohibited zones is divided into $\left(n_{i}+1\right)$ disjoint operating sub-regions. The total number of decision sub-spaces caused by that division may be counted as follows:

$$
N=\prod_{i \in \omega}\left(n_{i}+1\right)
$$

Equation (8) shows that the total number of decision sub-spaces rises extremely quickly as the number of units with prohibited zones rises.

\section{The PROPOSED AlgorithM}

\section{A. The AIA}

In AIA [10], mimics these biological principles of clone generation, proliferation and maturation. The main steps of AIS based on clonal selection principle are activation of antibodies, proliferation and differentiation on the encounter of cells with antigens, maturation by carrying out affinity maturation process, eliminating old antibodies to maintain the diversity of antibodies and to avoid premature convergence, selection of those antibodies whose affinities with the antigen are greater.

In order to emulate AIA in optimization, the antibodies and affinity are taken as the feasible solutions and the objective function respectively.

\section{B. The $M U$}

Michalewicz et al. [12] surveyed and compared several constraint-handling techniques used in evolutionary algorithms. Among these techniques, the penalty function method is one of the most popularly used to handle constraints. In this method, the objective function includes a penalty function that is composed of the squared or absolute constraint violation terms. Powell [13] noted that classical optimization methods include a penalty function have certain weaknesses that become most serious when penalty parameters are large. More importantly, large penalty parameters ill condition the penalty functions so that obtaining a good solution is difficult. However, if the penalty parameters are too small, the constraint violation does not contribute a high cost to the penalty function. Accordingly, choosing appropriate penalty parameters is not trivial. Herein, the MU [14] is introduced to handle this constrained optimization problem. Such a technique can overcome the ill conditioned property of the objective function.

Considering the nonlinear problem with general constraints as follows:

$$
\begin{array}{cc} 
& \min _{x} F(x) \\
\text { subject to } \quad & h_{k}(x)=0, \quad k=1, \ldots, m_{e} \\
& g_{k}(x) \leq 0, \quad k=1, \ldots, m_{i}
\end{array}
$$

where $h_{k}(x)$ and $g_{k}(x)$ stand for equality and inequality constraints, respectively.

The augmented Lagrange function (ALF) [13] for constrained optimization problems is defined as:

$$
\begin{aligned}
& L_{a}(x, v, v)=f(x)+ \\
& \sum_{k=1}^{m_{e}} \alpha_{k}\left\{\left[h_{k}(x)+v_{k}\right]^{2}-v_{k}^{2}\right\}+\sum_{k=1}^{m_{i}} \beta_{k}\left\{\left\langle g_{k}(x)+v_{k}\right\rangle_{+}^{2}-v_{k}^{2}\right\}
\end{aligned}
$$

where $\alpha_{k}$ and $\beta_{k}$ are the positive penalty parameters, and the corresponding Lagrange multipliers $v=\left(v_{1}, \ldots, v_{m_{e}}\right)$ and $\mathrm{v}=\left(\mathrm{v}_{1}, \ldots, \mathrm{v}_{m_{i}}\right) \geq 0$ are associated with equality and inequality constraints, respectively.

The contour of the ALF does not change shape between generations while constraints are linear. Therefore, the contour of the ALF is simply shifted or biased in relation to the original objective function, $f(x)$. Consequently, small penalty parameters can be used in the MU. However, the shape of contour of La is changed by penalty parameters while the constraints are nonlinear, demonstrating that large penalty parameters still create computational difficulties. Adaptive penalty parameters of the MU are employed to alleviate the above difficulties, and Table I presents computational procedures of the MU. More details of the MU have shown in [15]. 
TABLE I: COMPUTATIONAL PROCEDURES OF THE MU

Step 1. Set the initial iteration $l=0$. Set initial multiplier, $v_{k}^{l}=v_{k}^{0}=0, k=1, \ldots, m_{e}, v_{k}^{l}=v_{k}^{0}=0, k=1, \ldots, m_{i}$, and the initial penalty parameters, $\alpha_{k}>0, k=1, \ldots, m_{e}$ and $\beta_{k}>0, k=1, \ldots, m_{i}$. Set tolerance of the maximum constraint violation, $\varepsilon_{k}$ (e.g. $\varepsilon_{k}=10^{32}$ ), and the scalar factors, $\omega_{1}>1$ and $\omega_{2}>1$.

Step 2. Use a minimization solver, e.g. AIA, to solve $L_{a}\left(x, v^{l}, v^{l}\right)$. Let $x_{b}^{l}$, be a minimum solution to the problem $L_{a}\left(x, v^{l}, v^{l}\right)$.

Step 3. Evaluate the maximum constraint violation as $\hat{\varepsilon}_{k}=\max \left\{\max _{k}\left|h_{k}\right|, \max _{k} \mid \max \left(g_{k},-v_{k}\right)\right\}$, and establish the following sets of equality and inequality constraints whose violations have not been improved by the factor $\omega_{1}$ :

$$
\begin{aligned}
& I_{E}=\left\{k:\left|h_{k}\right|>\frac{\varepsilon_{k}}{\omega_{1}}, \quad k=1, \cdots, m_{e}\right\} \\
& I_{I}=\left\{k:\left|\max \left(g_{k}, \quad-v_{k}\right)\right|>\frac{\varepsilon_{k}}{\omega_{1}}, \quad k=1, \cdots, m_{i}\right\}
\end{aligned}
$$

Step 4. If $\hat{\varepsilon}_{k} \geq \varepsilon_{k}$, let $\alpha_{k}=\omega_{2} \alpha_{k}$ and $v_{k}^{l+1}=v_{k}^{l} / \omega_{2}$ for all $k \in I_{E}$, let $\beta_{k}=\omega_{2} \beta_{k}$ and $v_{k}^{l+1}=v_{k}^{l} / \omega_{2}$ for all $k \in I_{I}$, and go to step7. Otherwise, go to step 5 .

Step 5. Update the multipliers as follows:

$$
\begin{gathered}
v_{k}^{l+1}=h_{k}\left(x_{b}^{l}\right)+v_{k}^{l} \\
v_{k}^{l+1}=\left\langle g_{k}\left(x_{b}^{l}\right)+v_{k}^{l}\right\rangle_{+}=v_{k}^{l}+\max \left\{g_{k}\left(x_{b}^{l}\right),-v_{k}^{l}\right\}
\end{gathered}
$$

Step 6. If $\hat{\varepsilon}_{k} \leq \varepsilon_{k} / \omega_{1}$, let $\varepsilon_{k}=\hat{\varepsilon}_{k}$ and go to step 7 . Otherwise, let $\alpha_{k}=\omega_{2} \alpha_{k}$ and $v_{k}^{l+1}=v_{k}^{l} / \omega_{2}$ for all $k \in I_{E}$, and let $\beta_{k}=\omega_{2} \beta_{k}$ and $v_{k}^{l+1}=v_{k}^{l} / \omega_{2}$ for all $k \in I_{I}$. Let $\varepsilon_{k}=\hat{\varepsilon}_{k}$ and go to step 7 .

Step 7. If the maximum iteration reaches, stop. Otherwise, repeat steps 2 to 6 .

Fig. 1 displays the flow chart of the proposed algorithm, which has two iterative loops. The ALF is used to obtain a minimum value in the inner loop with the given penalty parameters and multipliers, which are then updated in the outer loop toward producing an upper limit of $L_{a}$. When both inner and outer iterations become sufficiently large, the ALF converges to a saddle-point of the dual problem [15]. Advantages of the proposed AIA-MU are that the AIA efficiently searches the optimal solution in the economic dispatch process and the MU effectively tackles system constraints.

\section{SYSTEM SIMULATIONS}

This section investigates two examples to illustrate the effectiveness of the proposed algorithm with respect to the quality of the solution obtained. The first example compares the proposed AIA-MU with the previous methods in terms of production cost for a 5-unit system. The second example compares the AIA-MU with the previous methods in terms of production cost for a 15-unit system, in which four units (Units 2, 5, 6 and 12) have POZ.

The proposed AIA-MU was directly coded using real values, and the computation was implemented on a personal computer (P5-3.0 GHz) in FORTRAN-90. Setting factors utilized in these examples were as follows: the population size $N_{p}$ was set to 5 for the proposed AIA-MU, and iteration numbers of the outer loop and inner loop were set to (outer, inner) as $(50,5000)$ for examples of 1 and 2 . For most setting of the parameters, the proposed method is able to converge satisfactorily.

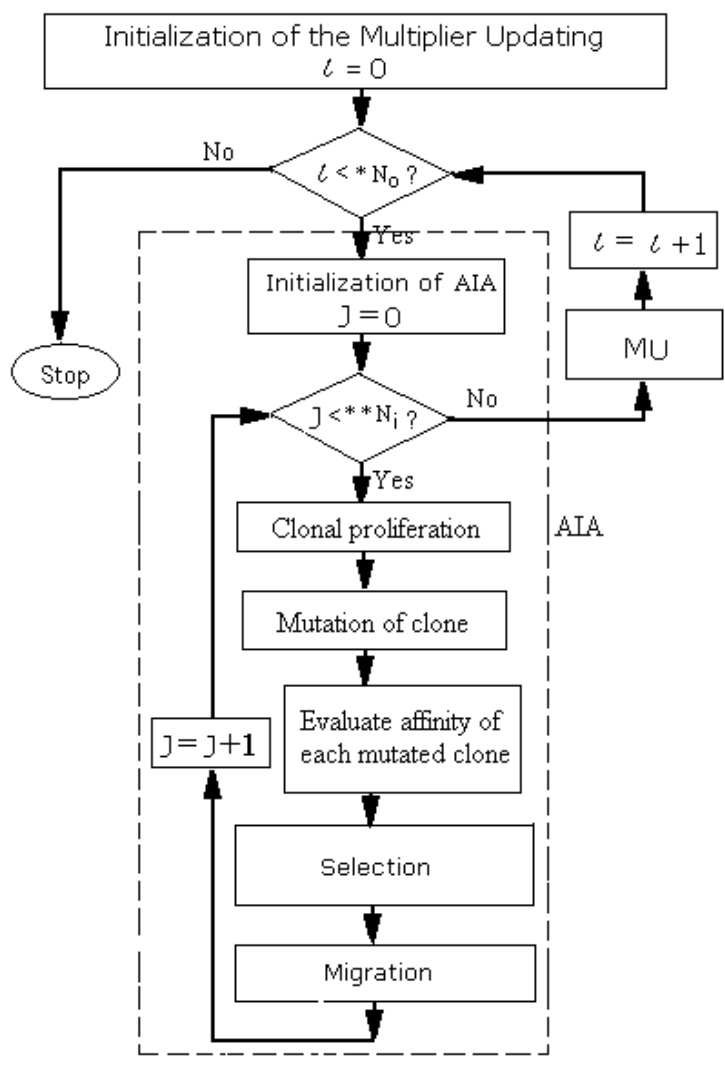

Fig. 1 The flow chart of the AIA-MU

$* \mathrm{~N}_{0}$ : maximum number of iterations of outer loop

$* * \mathrm{~N}_{\mathrm{i}}$ : maximum number of iteartions of inner loop

Fig. 1. The flow chart of thr AIA-MU.

\section{A. Example 1}

This example system has five on-line units with the following input-output cost functions:

$$
F_{i}\left(P_{i}\right)=350+8 P_{i}+0.01 P_{i}^{2}+1 \cdot 10^{-6} P_{i}^{3} \$ / h
$$

where $i=1, \ldots, 5$. The operating limits are $120 \mathrm{MW}<P_{i}<$ 450MW for $i=1,2, \ldots, 5$. Units 1,2 and 3 have POZ as defined in [2], these zones result in a non-convex decision space composed of 27 convex sub-spaces. The system load demand $P_{D}$ and spinning reserve $S_{R}$ are $1175 \mathrm{MW}$ and 100 MW, respectively.

For comparison, Table II lists the computational results of the proposed AIA-MU, the $\lambda-\delta$ Method [2], and the EP Method [6]. The total cost obtained by the AIA-MU is satisfactory compared with that obtained by the $\lambda-\delta$ method [2] and the EP method [6].

TABLE II: COMPUTATIONAL RESUlts OF THE PREVIOUS METHODS AND THE PROPOSED IGAMUM FOR EXAMPLE 1

\begin{tabular}{cccc}
\hline Items & $\lambda-\delta$ Method [2] & EP Method [6] & AIA-MU \\
\hline $\mathrm{P}_{1}(\mathrm{MW})$ & 238.33 & 240.00 & 238.13 \\
$\mathrm{P}_{2}(\mathrm{MW})$ & 210.00 & 210.00 & 210.00 \\
$\mathrm{P}_{3}(\mathrm{MW})$ & 250.00 & 250.00 & 250.00 \\
$\mathrm{P}_{4}(\mathrm{MW})$ & 238.33 & 223.07 & 238.46 \\
$\mathrm{P}_{5}(\mathrm{MW})$ & 238.33 & 251.93 & 238.41 \\
Total power $(\mathrm{MW})$ & $1,174.99$ & $1,175.00$ & $1,175.00$ \\
Total cost $(\$ / \mathrm{h})$ & $11,492.51$ & $11,493.23$ & $11,492.50$ \\
\hline
\end{tabular}

Fan et al. [2] defined a small and advantageous set of decision space with respect to the system demand, used an 
algorithm to determine the most advantageous space, and then utilized the $\lambda-\delta$ iterative method to find the feasible optimal dispatch solution. For infeasible solutions, they re-dispatch the units using some heuristic rules to probe the neighborhood for feasibility. The AIA-MU combines the AIA and the MU. The AIA can efficiently search and actively explore solutions, the MU avoids deforming the augmented Lagrange function and resulting in difficulty of solution searching. Unlike the method [2], it requires neither the decomposition of the non-convex decision space nor the determination of the advantageous set of decision space before solving via this conventional approach.

\section{B. Example 2}

To further demonstrate the effectiveness of the proposed method, a larger practical system of units with POZ having non-convex cost functions and spinning reserve was addressed in this example, which is identical to that used by Lee et al. [1]. The remaining units will contribute with regulating reserves [16]. This system supplies a 2650MW load demand with 200MW as spinning reserve. This system has 15 on-line units supplying a system demand of 2650MW. Among these dispatching generators, units 2, 5 and 6 have three POZ, and unit 12 has two POZ, forming 192 decision sub-spaces for this realistic system. The implementation of this example can be represented as follows:

$$
\begin{gathered}
L_{a}(x, v, v)=f(x)+\alpha_{1}\left\{\left[h_{1}(x)+v_{1}\right]^{2}-v_{1}^{2}\right\} \\
+\sum_{k=1}^{5} \beta_{k}\left\{\left\langle g_{k}(x)+v_{k}\right\rangle_{+}^{2}-v_{k}^{2}\right\}
\end{gathered}
$$

objective : $\min _{x=\left(P_{1}, P_{2}, \ldots P_{15}\right)} f(x)=\sum_{i=1}^{15} F_{i}\left(P_{i}\right)$

subject to $h_{1}: \quad \sum_{i=1}^{15} P_{i}-P_{d}-P_{L}=0$

$$
\begin{array}{llll}
g_{1}: P_{2}^{150} \leq P_{2} \leq P_{2,1}^{185}, \text { or } & P_{2,1}^{225} \leq P_{2} \leq P_{2,2}^{305}, \text { or } & P_{2,2}^{335} \leq P_{2} \leq P_{2,3}^{420}, \text { or } & P_{2,3}^{450} \leq P_{2} \leq P_{2}^{455} \\
g_{2}: P_{5}^{105} \leq P_{5} \leq P_{5,1}^{180}, \text { or } & P_{5,1}^{200} \leq P_{5} \leq P_{5,2}^{260} \text {, or } & P_{5,2}^{335} \leq P_{5} \leq P_{5,3}^{390} \text { or } & P_{5,3}^{420} \leq P_{5} \leq P_{5}^{470} \\
g_{3}: P_{6}^{135} \leq P_{6} \leq P_{6,1}^{230} \text {, or } & P_{6,1}^{255} \leq P_{6} \leq P_{6,2}^{365} \text {,or } & P_{6,2}^{395} \leq P_{6} \leq P_{6,3}^{430} \text {,or } & P_{6,3}^{455} \leq P_{6} \leq P_{6}^{460} \\
g_{4}: P_{12}^{20} \leq P_{12} \leq P_{12,1}^{30}, \text { or } & P_{12,1}^{55} \leq P_{12} \leq P_{12,2}^{65}, \text { or } & P_{12,2}^{75} \leq P_{12} \leq P_{12}^{80} \\
g_{5}: S_{R}-\sum_{i=1}^{15} S_{i} \leq 0
\end{array}
$$

\begin{tabular}{|c|c|c|c|c|c|}
\hline Items & $\begin{array}{l}\lambda-\delta \\
{[1]}\end{array}$ & $\begin{array}{l}\lambda-\delta \\
{[2]}\end{array}$ & $\begin{array}{c}\text { Hopfield } \\
\text { [3] }\end{array}$ & $\begin{array}{c}\text { Hopfield } \\
\text { [4] }\end{array}$ & AIA-MU \\
\hline$P_{l}(\mathrm{MW})$ & 450 & 450.0 & 449.4 & 454.6976 & 449.9995 \\
\hline$P_{2}(\mathrm{MW})$ & 450 & 450.0 & 450 & 454.6976 & 450.0001 \\
\hline$P_{3}(\mathrm{MW})$ & 130 & 130.0 & 130 & 129.3512 & 130.0000 \\
\hline$P_{4}(\mathrm{MW})$ & 130 & 130.0 & 130 & 129.3512 & 130.0000 \\
\hline$P_{5}(\mathrm{MW})$ & 335 & 335.0 & 335 & 244.9966 & 335.0001 \\
\hline$P_{6}(\mathrm{MW})$ & 455 & 455.0 & 455 & 459.6919 & 455.0003 \\
\hline$P_{7}(\mathrm{MW})$ & 465 & 465.0 & 464.9 & 464.6916 & 465.0000 \\
\hline$P_{8}(\mathrm{MW})$ & 60 & 60.0 & 60 & 60.0938 & 60.0000 \\
\hline$P_{9}(\mathrm{MW})$ & 25 & 25.0 & 25 & 25.0496 & 25.0000 \\
\hline$P_{10}(\mathrm{MW})$ & 20 & 20.0 & 20 & 89.1023 & 20.0000 \\
\hline$P_{l I}(\mathrm{MW})$ & 20 & 20.0 & 20 & 20.0338 & 20.0000 \\
\hline$P_{12}(\mathrm{MW})$ & 55 & 55.0 & 55 & 63.1815 & 55.0002 \\
\hline$P_{13}(\mathrm{MW})$ & 25 & 25.0 & 25 & 25.0527 & 25.0000 \\
\hline$P_{14}(\mathrm{MW})$ & 15 & 15.0 & 15 & 15.0044 & 15.0000 \\
\hline$P_{15}(\mathrm{MW})$ & 15 & 15.0 & 15 & 15.0044 & 15.0000 \\
\hline$T P(\mathrm{MW})$ & 2650 & 2650 & 2649.3 & 2650.0002 & 2650.0000 \\
\hline sum_Si (MW) & 235 & 235.0 & 235.7 & 231.8996 & 235.0005 \\
\hline$T C(\$ / \mathrm{h})$ & $32,549.8$ & $32,544.99$ & $32,538.4$ & 32,568 & $32,544.9705$ \\
\hline CPU_time(s) & - & - & - & - & 4.88 \\
\hline
\end{tabular}

\begin{tabular}{|c|c|c|c|c|c|}
\hline Items & $\begin{array}{l}\text { GA } \\
{[17]}\end{array}$ & $\begin{array}{c}\text { SA } \\
{[17]}\end{array}$ & $\begin{array}{c}\mathrm{HDE} \\
{[17]} \\
\end{array}$ & $\begin{array}{c}\text { VSHDE } \\
{[17]} \\
\end{array}$ & AIA-MU \\
\hline$P_{l}(\mathrm{MW})$ & 415.85 & 413.22 & 455.00 & 454.74 & 455.0000 \\
\hline$P_{2}(\mathbf{M W})$ & 450.00 & 167.67 & 336.16 & 424.96* & 450.0000 \\
\hline$P_{3}(\mathrm{MW})$ & 111.87 & 99.91 & 128.39 & 129.87 & 130.0000 \\
\hline$P_{4}(\mathrm{MW})$ & 121.46 & 21.36 & 129.92 & 129.99 & 130.0000 \\
\hline$P_{5}(\mathbf{M W})$ & 340.84 & 449.20 & 420.00 & $397.36^{*}$ & 335.0000 \\
\hline$P_{6}(\mathrm{MW})$ & 455.00 & 296.28 & 418.73 & 500.00 & 460.0000 \\
\hline$P_{7}(\mathrm{MW})$ & 333.78 & 360.15 & 443.03 & 464.75 & 430.1175 \\
\hline$P_{8}(\mathrm{MW})$ & 81.84 & 287.99 & 60.00 & 60.00 & 60.0000 \\
\hline$P_{9}(\mathrm{MW})$ & 115.48 & 155.27 & 41.42 & 25.89 & 25.0000 \\
\hline$P_{10}(\mathrm{MW})$ & 60.59 & 138.81 & 107.17 & 20.75 & 84.5692 \\
\hline$P_{11}(\mathrm{MW})$ & 31.78 & 47.85 & 20.00 & 20.00 & 20.0000 \\
\hline$P_{12}(\mathrm{MW})$ & 55.00 & 76.97 & 79.59 & 75.86 & 80.0000 \\
\hline$P_{13}(\mathrm{MW})$ & 70.39 & 81.91 & 36.98 & 25.06 & 25.0000 \\
\hline$P_{14}(\mathrm{MW})$ & 26.22 & 52.94 & 22.02 & 15.13 & 15.0000 \\
\hline$P_{15}(\mathrm{MW})$ & 36.34 & 52.95 & 15.00 & 15.00 & 15.0000 \\
\hline$T P(\mathrm{MW})$ & $2,704.46$ & $2,702.49$ & $2,713.40$ & $2,719.35$ & $2,714.6867$ \\
\hline$P_{L}(\mathrm{MW})$ & 56.45 & 52.49 & 63.41 & 69.35 & 64.6867 \\
\hline $\begin{array}{c}\text { sum_Si } \\
\text { (MW) }\end{array}$ & 307.86 & 218.90 & 246.65 & 230.53 & 264.8825 \\
\hline$T C(\$ / \mathrm{h})$ & $\begin{array}{c}33,538.2 \\
7\end{array}$ & $\begin{array}{c}34,174.4 \\
5\end{array}$ & $\begin{array}{c}33,343.3 \\
7\end{array}$ & $\begin{array}{c}\text { 33,282.17 } \\
\#\end{array}$ & $\begin{array}{c}33,259.305 \\
5\end{array}$ \\
\hline $\begin{array}{c}C P U_{-} \text {time }(\mathrm{s} \\
)\end{array}$ & - & - & - & - & 5.02 \\
\hline
\end{tabular}

TABLE III: COMPARED RESULTS WITHOUT LOSS OF THE PREVIOUS METHODS AND THE AIA-MU
TABLE IV: COMPARED RESULTS CONSIDERING LOSS OF THE PREVIOUS METHODS AND THE AIA-MU 
This complex optimization problem contains one objective function with fifteen variable parameters, $\left(P_{1}, P_{2}, \ldots, P_{15}\right)$, one equality constraint, $\left(h_{1}\right)$ and five inequality constraints, since four units have the POZ, $\left(g_{1}\right.$ to $\left.g_{4}\right)$, and the spinning reserve constraint $\left(g_{5}\right)$.

Table III lists five algorithms of this problem with POZ and spinning reserve constraints obtained by two $\lambda-\delta$ methods, two Hopfield methods and the proposed AIAE-MU. Computational results demonstrate that the proposed method is a little better than the two $\lambda-\delta$ methods and the Hopfield method. Even though the Hopfield method has a little less total cost than the proposed method, but its total generated power is $2649.3 \mathrm{MW}$, which is $0.7 \mathrm{MW}$ less than the system load demand. In table III, the sum_S $S_{i}$ and $C P U \_$time stand the sum of spinning reserve and simulation time obtained from the method, respectively. Table III reveals that the proposed AIA-MU not only has the lowest total cost (TC) of all methods tested, but also generates the exact total power (TP) for the system constraints of (14) and (15), showing that the proposed algorithm is more effective than other methods for the practical PED problem with POZ.

Moreover, Table IV shows compared results obtained with the previous variable scaling HDE (VSHDE) [17], the GA [17], simulated annealing (SA) [17] and the propose AIA-MU for the system with the transmission loss. The result obtained by the VSHDE method is an infeasible solution, because generations of Unit 2 and Unit 5, $\left(P_{2}\right.$ and $\left.P_{5}\right)$, are located in POZ, respectively. The proposed algorithm also yields better solution quality than other methods in the PED problem considering POZ.

\section{CONCLUSIONS}

An efficient method for solving the optimal PED problem considering POZ was proposed. The proposed approach integrates the AIA and the MU, showing that the proposed algorithm has the following merits: 1) ease of implementation; 2) applicability to non-convex fuel cost functions of the POZ; 3) better effectiveness than the previous method, and 4) the need for only a small population. System simulations have shown that the proposed approach has the advantages mentioned above for solving optimal PED problems of the system with POZ.

\section{ACKNOWLEDGMENT}

Financial support given to this research by the National Science Council, Taiwan, ROC. under Grant No. NSC100-2632-E-252-001-MY3 is greatly appreciated.

\section{REFERENCES}

[1] F. N. Lee and A. M. Breipohl, "Reserve constrained economic dispatch with prohibited operating zones," IEEE Trans. on Power System, vol. 8 , no. 1, pp. 246-254, 1993.

[2] J. Y. Fan and J. D. McDonald, "A practical approach to real time economic dispatch considering unit's prohibited operating zones," IEEE Trans. on Power System, vol. 9, no. 4, pp. 1737-1743, 1994.

[3] C. T. Su and G. J. Chiou, "A Hopfield network approach to economic dispatch with prohibited operating zones," in Proc. the IEEE International Conf. on Energy Management and Power Delivery, 1995, pp. 382-387.

[4] T. Yalcinoz, H. Altun, and U. Hasan, "Constrained economic dispatch with prohibited operating zones: a Hopfiled neural network approach," in Proc. IEEE 10th Mediterranean Electrotechnical Conf. MEleCon, II, 2000, pp. 570-573.

[5] S. O. Orero and M. R. Irving, "Economic dispatch of generators with prohibited operating zones: a genetic algorithm approach," IEE Proc-Gener. Transm. Distrib., vol. 143, issue 6, pp. 529-534, 1996.

[6] T. Jayabarathi, G. Sadasivam, and V. Ramachandran, "Evolutionary programming based economic dispatch of generators with prohibited operating zones," Electric Power Systems Research, vol. 52, pp. 261-266, 1999.

[7] A. Pereira-Neto, C. Unsihuay, and O. R. Saavedra, "Efficient evolutionary strategy optimisation procedure to solve the nonconvex economic dispatch problem with generator constraints," in Proc. IEE Proc.-Gener. Transm. Distrib., pp. 653-660, 2005, vol. 152, issue 5.

[8] K. T. Chaturvedi, M. Pandit, and L. Srivastava, "Particle swarm optimization with time varying acceleration coefficients for non-convex economic power dispatch," Electrical Power and Energy Systems, vol. 31, pp. 249-257, 2009.

[9] S. Hemamalini and S. P. Simon, "Dynamic economic dispatch using artificial immune system for units with valve-point effect," Electrical Power and Energy Systems, vol. 33, pp. 868-874, 2011.

[10] M. Basu, "Artificial immune system for combined heat and power economic dispatch," Electrical Power and Energy Systems, vol. 43, pp. $1-5,2012$.

[11] A. J. Wood and B. F. Wollenberg, Power Generation Operation, and Control, New York, John Wiley \& Sons, 1996.

[12] Z. Michalewicz and M. Schoenauer, "Evolutionary algorithms for constrained parameter optimization problems," Evolutionary Computation, vol. 4, no. 1, pp. 1-32, 1996.

[13] M. J. D. Powell, "Algorithms for nonlinear constraints that use Lagrangian function," Math. Programming, vol. 14, pp. 224-248, 1978.

[14] C. L. Chiang, "Hybrid Differential Evolution for Power Economic Dispatch Problems Considering the incorporated cost Model," International Journal Applied Mechanics and Materials, vol. 302, pp. 782-786, 2013.

[15] C. L. Chiang, C. T. Su, and F. S. Wang, "Augmented lagrangian method for evolutionary optimization of mixed-integer nonlinear constrained problems," Intern. Math. J., vol. 2, no. 2, pp. 119-154, 2002.

[16] W. M. Lin, F. S. Cheng and M. T. Tsay, "Nonconvex economic dispatch by integrated artificial intelligence," IEEE Trans. on Power System, vol. 16, no. 2, pp. 307-311, 2001.

[17] J. P. Chiou, "Variable scaling hybrid differential evolution for large-scale economic dispatch problems," Electric Power Systems Research, vol. 77, pp. 212-218, 2007.

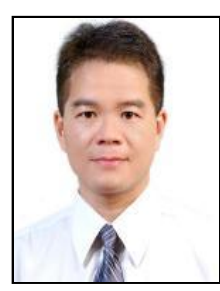

C. L. Chiang received his M.S. degree from Automatic Control Engineering, Feng Chia University, Taichung, Taiwan in 1991, and Ph.D. degree from Institute of Electrical Engineering, National Chung Cheng University, Chia-Yi, Taiwan in 2004.

He is now a professor of Nan Kai University of Technology, Nan-Tou, Taiwan, ROC. His research interests are in the control theory, applications of the optimization, evolutionary algorithms and power economic dispatch. 